\title{
Nematic-Smectic Transition under Confinement in Liquid Crystalline Colloidal Shells
}

\author{
Hsin-Ling Liang, ${ }^{1}$ Stefan Schymura, ${ }^{1}$ Per Rudquist, ${ }^{2}$ and Jan Lagerwall ${ }^{1,3, *}$ \\ ${ }^{1}$ Martin-Luther University Halle-Wittenberg, Institute of Chemistry - Physical Chemistry, \\ Von-Danckelmann-Platz, 4, 06120 Halle, Germany \\ ${ }^{2}$ Chalmers University of Technology, Department for Microtechnology and Nanoscience, 41269 Göteborg, Sweden \\ ${ }^{3}$ Seoul National University, Graduate School of Convergence Science and Technology, Suwon-si, Gyeonggi-do, Korea 443-270
}

(Received 1 February 2011; published 15 June 2011)

\begin{abstract}
We carry out the first study of smectic liquid crystalline colloidal shells and investigate how their complex internal structure depends on the director configuration in the nematic phase, preceding the smectic phase on cooling. Differences in the free energy cost of director bend and splay give an initial skewed distribution of topological defects in the nematic phase. In the smectic phase, the topological and geometrical constraints of the spherical shell imposed on the developing 1D quasi-long-range order create a conflict that triggers a series of buckling instabilities. Two different characteristic defect patterns arise, one driven by the curvature of the shell, the other by the strong nonuniformities in the director field in the vicinity of the topological defects.
\end{abstract}

DOI: 10.1103/PhysRevLett.106.247801

PACS numbers: 61.30.Jf, 61.30.Pq, 64.70.mj

Colloidal liquid crystalline shells have recently been introduced as a new potent concept in soft condensed matter physics. They display phenomena that are fascinating from a fundamental physics point of view [1-7] and they also hold promise for innovative applications [8]. The key feature of these shells is the presence of topological defects in the director field $\mathbf{n}(\mathbf{r})$ (the director $\mathbf{n}$ indicates the average molecule orientation). For thin symmetric planar-aligned shells of the simplest liquid crystal type, the nematic $(N)$, one expects an arrangement of four $s=1 / 2$ defects on a tetrahedron or on a great circle, depending on the details of the elastic constants [5]. The former configuration has potential for the generation of colloidal crystals with diamond geometry [8]: because the defects constitute points of localized breakdown of order they can act as attractors for additives [9]. Adding molecules designed for giving the shells interaction capacity, these would thus be anchored into a tetrahedral geometry, endowing the shells with directed bonding analogous to $s p^{3}$-hybridized carbon. Other phases should stabilize different numbers and configurations of defects, giving a means of tuning the valence via phase transitions, and the configuration may be more stable than in the highly fluid nematic phase.

So far attention has been devoted to the nematic phase, exhibiting long-range orientational order but no positional order. As we show in this Letter, the addition of the 1D positional order characteristic of smectic phases [10] dramatically changes the structuring within the shell. The constraints imposed by the layered molecule organization of smectics affects the defect configuration and leads to a more complex internal structure, giving new possibilities and different stable states compared to nematics. An understanding of the ordering processes in smectic shells is thus of fundamental physics interest but also relevant for the goal to apply the shells. We investigate for the first time the development of smectic structure in a planar-aligned shell geometry upon cooling from the $N$ to the smectic- $A$ (SmA) phase. We find a strong interplay of the shell geometry and the smectic ordering process, leading to a series of buckling instabilities that eventually produce two different types of regular texture.

The shells are produced using a microfluidics setup adapted from the design of Weitz and coworkers [11], previously described in [12]. An inner phase consisting of $1 \mathrm{wt} \%$ poly(vinyl alcohol) (PVA) in a 1:1 volume ratio water/glycerol mixture is co-flown inside the liquid crystalline material (4-cyano-4-octylbiphenyl, 8CB: SmA 33.5 $N 41.5$ Iso. $/{ }^{\circ} \mathrm{C}$ ), and this composite fluid is flow focused by an outer aqueous phase containing $5 \mathrm{wt} \%$ of the surfactant Pluronics F127. By mounting the system in a heated stage $\left(60^{\circ} \mathrm{C}\right) 8 \mathrm{CB}$ is kept in its isotropic state throughout the preparation process. The inner phase has a density slightly above that of $8 \mathrm{CB}$; hence, the inner droplet (radius $a$ ) sinks to the bottom of each shell (radius $R$ ), making it thicker at the top and thinner at the bottom, cf. Fig. 1. While PVA ensures strong planar alignment on the shell inside, F127 allows planar as well as homeotropic alignment [12]. In the thin shells considered here (a few $\mu \mathrm{m}$ average thickness $\bar{h}=R-a$ ) the inside PVA dominates,

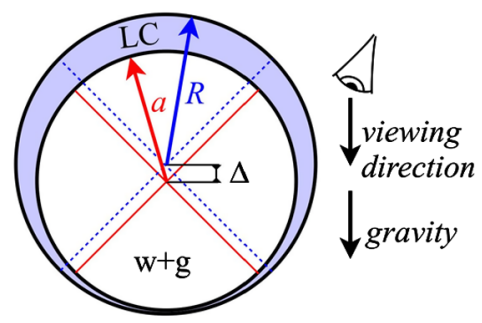

FIG. 1 (color online). Geometry of the liquid crystal (LC) shell and the water/glycerol $(w+g)$ inner phase. 
imposing planar alignment throughout the shell. After production the shells are filled into a flat capillary which is sealed and placed in a hot stage mounted on a polarizing microscope.

Figure 2 shows a series of polarizing microscopy photos of a shell that had been cooled from the isotropic to the nematic phase, developing the texture in panel $a$. Four $s=$ $1 / 2$ defects are gathered close to the bottom thinnest part of the shell. As explained by Fernandez-Nieves et al. [1] and elaborated by Lopez-Leon et al. [2] this local defect clustering occurs in asymmetric shells $(\Delta \neq 0$ in Fig. 1$)$, provided that the normalized average thickness $u=\bar{h} / R$ is greater than a threshold value $u_{0}$, in order to reduce the length of the defect cores and thereby minimize the energy. In our case $u>u_{0}$ apparently holds.

What has not previously been noted is that the defects are typically not organized symmetrically around the thinnest part of the shell but they adopt a skewed configuration with the pair labeled 2 and 4 in Fig. 2 separated by about twice the distance of 1 and 3. This arrangement can be understood by recognizing that the defects are not quite identical. A basic polarization optics analysis of the images with respect to bright and dark areas allows us to deduce $\mathbf{n}(\mathbf{r})$ to within two possible configurations differing by a rotation of $90^{\circ}$. The remaining degeneracy can be lifted using standard birefringent compensators in the microscope, or by studying the subsequent textural development

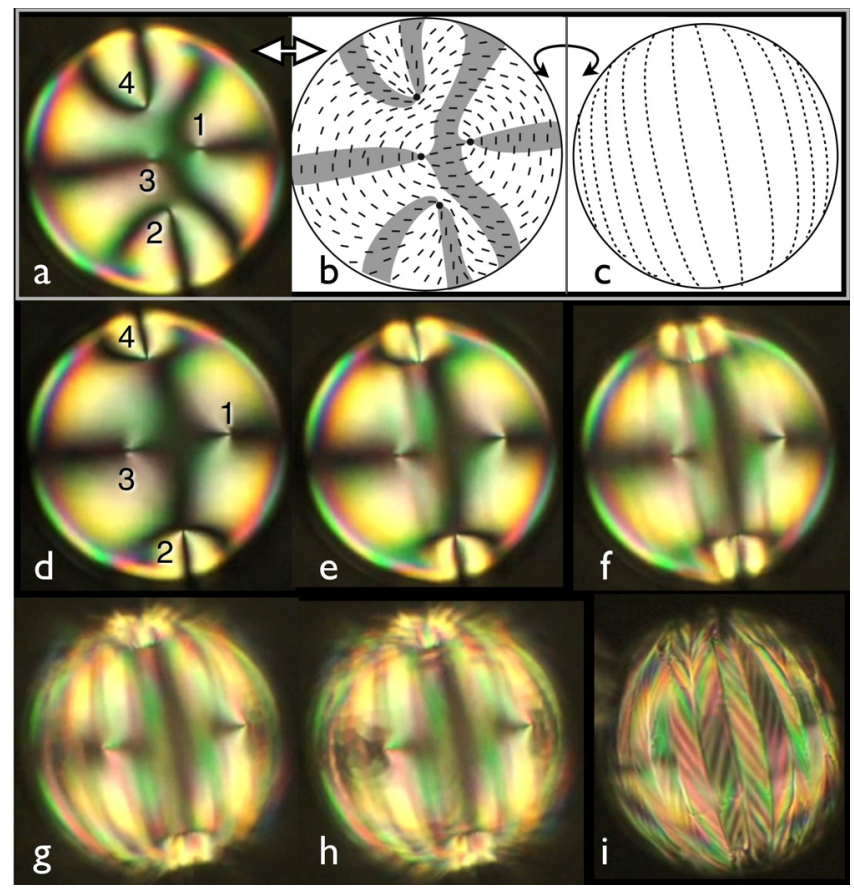

FIG. 2 (color online), $N$-SmA transition in a planar-aligned shell ( $R \approx 100 \mu \mathrm{m}, \bar{h} \approx 4 \mu \mathrm{m})$. In (a)-(h) the focus is on the thin shell side, where the defects are concentrated, thus the initial signs of the transition (e)-(g) - starting on the thick side-are blurry. In (i) the focus is on the thick side. Drawings (b) and (c) sketch $\mathbf{n}(\mathbf{r})$ corresponding to (a) on the thin and thick sides, respectively. after cooling to SmA, where the layers develop perpendicular to $\mathbf{n}$. We can thus sketch $\mathbf{n}(\mathbf{r})$ in the shell as in Figs. 2(b) and 2(c). Figure 2(b) shows that defects 1 and 3 are connected primarily to the director bend whereas $\mathbf{n}$ is mainly splayed around 2 and 4 . The free energy density connected to deformations in $\mathbf{n}(\mathbf{r})$ is given by the OseenFrank expression:

$$
\begin{aligned}
G= & \frac{1}{2} K_{1}(\nabla \cdot \mathbf{n})^{2}+\frac{1}{2} K_{2}(\mathbf{n} \cdot \nabla \times \mathbf{n})^{2} \\
& +\frac{1}{2} K_{3}(\mathbf{n} \times \nabla \times \mathbf{n})^{2}
\end{aligned}
$$

where $K_{1}, K_{2}$, and $K_{3}$ are the elastic constants for splay, twist, and bend, respectively. Because bend has a greater energy penalty than splay $\left(K_{3} \approx 2 K_{1}\right)$ it is favorable to have the 1 and 3 defects as close to the thinnest part of the shell as possible, minimizing their core lengths more than for the less costly 2 and 4 defects.

On cooling towards the $\mathrm{N}$-SmA transition the defects move further apart, cf. Fig. 2(d). This is probably a sign of a slight reduction in shell asymmetry $\Delta$ close to SmA, making the clustering of defects less favorable. While it is tempting to think that the movement would be related to the divergence of elastic constants on approaching the smectic phase, it is actually only $K_{2}$ and $K_{3}$ that diverge, while the increase in $K_{1}$ is much less dramatic. Should variation in the elastic constants be the origin, the ratio between the defect pair distances should thus change, but it remains roughly unchanged until the phase transition. Moreover, the divergence of $K_{3}$ should in case of constant $\Delta$ drive the 1 and 3 defect pair towards the shell bottom to further minimize the core length (although a minimum distance must be maintained by the repulsion of like-signed defects), but the exact opposite happens. The conjectured decrease of $\Delta$ on approaching the phase transition needs to be verified in future investigations but it can be considered plausible since the energy penalty connected to the shell's curvature when a smectic state develops (to be discussed below) increases with shell thickness; hence, it could drive a flow from the thicker to the thinner part of the shell.

The $N$-SmA transition occurs first on the top, thicker side of the shell, which has no defects in $\mathbf{n}(\mathbf{r})$, cf. Fig. 2(c). It starts with the appearance of thin stripes running parallel to the original nematic director field $\mathbf{n}(\mathbf{r})_{N}$, as seen in Fig. 3 which shows photos of the very initial stage of the transition with focus on the thick shell side. Although the shell imaged here is larger and somewhat thicker than in Fig. 2 (hence the difference in color) the texture sequence is fundamentally the same. In Figs. 5(d) and 5(e) the stripe pattern can be seen also along the perimeter of a shell with dimensions comparable to that in Fig. 2. The bright-dark alternation between stripes reveals that the effective optic axis is rotated from the original $\mathbf{n}(\mathbf{r})_{N}$ orientation, in opposite senses in adjacent stripes. This modulation in the smectic phase shows that the layers are undulated, a common consequence of strain on the layers [13-15]. The strain appears because of the shell's curvature, which gives 

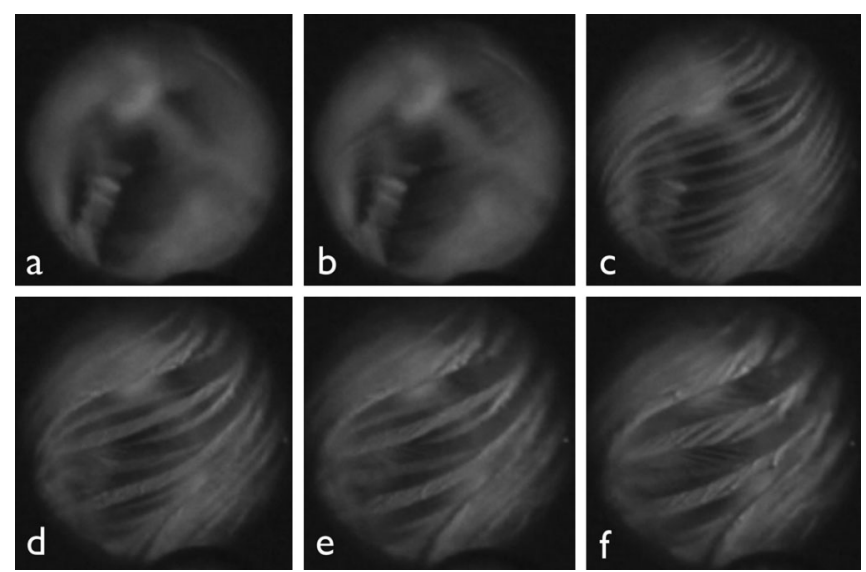

FIG. 3. Planar-aligned shell $(R \approx 350 \mu \mathrm{m}, \bar{h} \approx 10 \mu \mathrm{m})$ in its nematic phase (a) and during the very initial stage of the $N$-SmA transition (b-f). The focus is on the thicker side of the shell, where the transition starts.

a bend in $\mathbf{n}(\mathbf{r})$ - prohibited in SmA — and leads to a greater surface area of the outer than of the inner shell boundary, cf. Fig. 4. If $\mathbf{n}(\mathbf{r})$ would remain intact as $\mathbf{n}(\mathbf{r})_{N}$ after the transition, a greater number of layers would be needed at the outside than at the inside, requiring many costly dislocations [Fig. 4(a), top right]. The problem increases in severity the thicker the shell. However, if the layers buckle increasingly [Fig. 4(b) and bottom right of 4(a)] on approaching the shell's outer boundary, the interlayer distance $a_{1}$ measured along $\mathbf{n}(\mathbf{r})_{N}$ (retained only at the inner boundary) is greater than the smectic layer thickness $a_{0}$, thus accommodating the shell geometry with the condition of constant smectic layer thickness.
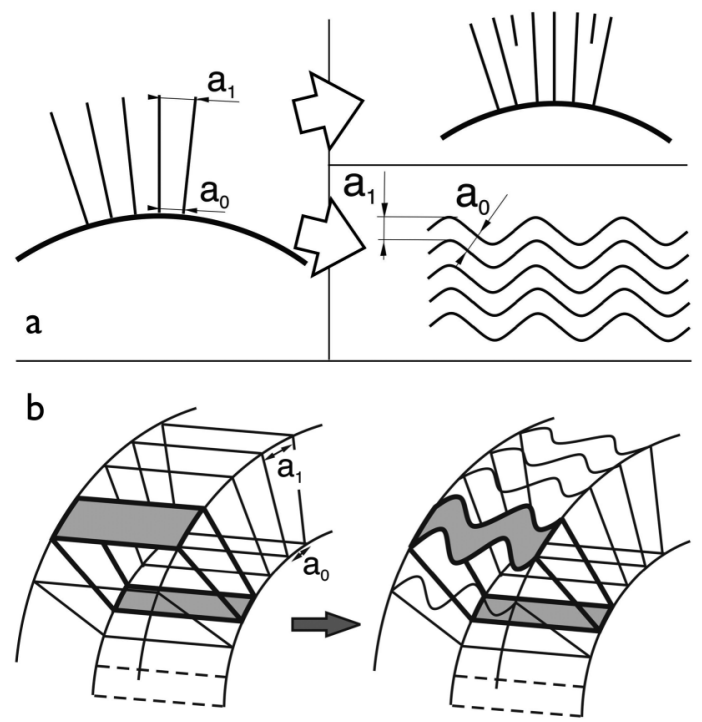

FIG. 4. (a) Smectic layering in a curved shell poses problems for retaining the smectic layer thickness, which can be solved by dislocations (upper path) or layer buckling (lower path). (b) Sketch of how the effective layer distance along a cut can increase from $a_{0}$ at the inside to $a_{1}>a_{0}$ at the outside through a gradually increasing layer undulation.
The distance $a_{1}$ increases as $a_{1}=a_{0} / \cos \alpha$, where $\alpha$ is the angle by which the layers twist locally, increasing continuously from zero at the shell inside to a limiting value $\alpha_{0}$ at the outside. The geometrical relation between the curvature, thickness and undulation amplitude allows us to estimate the shell thickness from the optic axis oscillations. Because of the dynamics of the system these are unfortunately very difficult to measure, but an estimate of about $\pm 15^{\circ}$ and a shell radius of $50 \mu \mathrm{m}$ for the shell in Fig. 2 results in a maximum shell thickness of $\sim 7 \mu \mathrm{m}$, in good agreement with the observed strong birefringence colors and average thickness $\bar{h} \approx 4 \mu \mathrm{m}$ determined by direct observation in polarized light.

The stripes run all around the thick shell side, extending over to the thin side toward their ends, as if they would be suspended between defects 2 and 4 in Figs. 2 and 5 [best seen in Figs. 5(d) and 5(e)]. Almost immediately after the pattern has been established the stripes start merging [Figs. 3(d) and 3(e)] into broader spherical lunes with quite sharp boundaries [Fig. 3(f)], again with alternating orientation of optic axis. This pattern is basically the equilibrium texture of the SmA phase in the shells studied here, cf. Fig. 2(i) and Figs. 5(h) and 5(i). In the latter case the shell is aligned in such a way with respect to the crossed polarizers that the alternating dark-bright character of the lunes is easily seen in panel $h$, whereas this alternation disappears upon removing the analyzer as in panel $i$.

Each lune also exhibits an internal periodic inclined ribbon pattern, giving a chevron character to the final

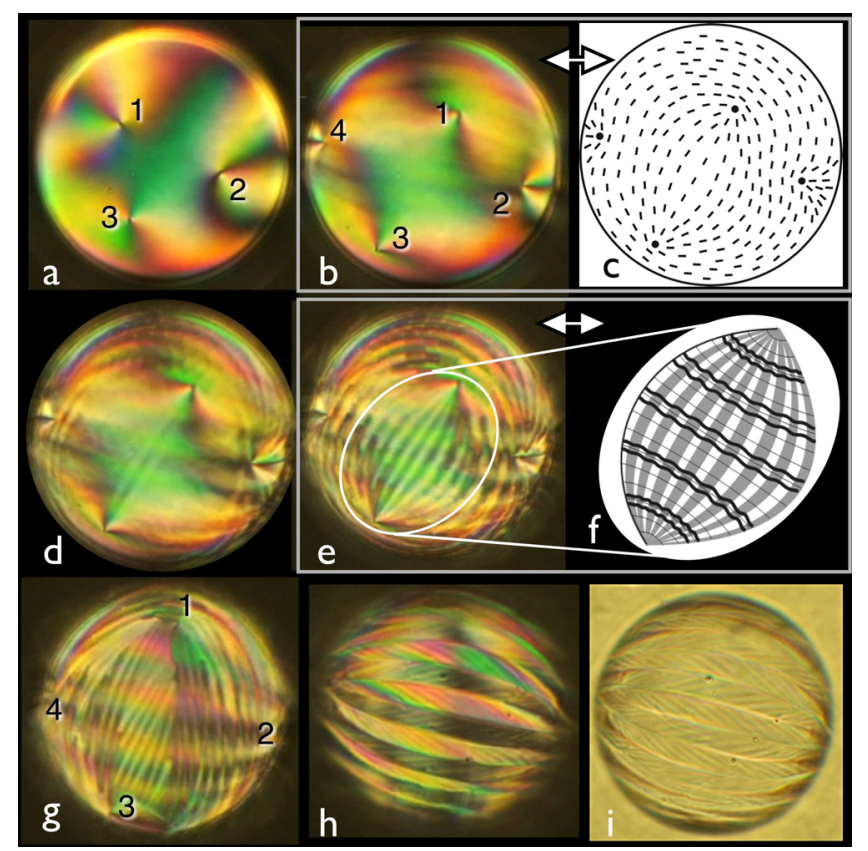

FIG. 5 (color online). $N$-SmA transition in a planar-aligned shell $(R \approx 100 \mu \mathrm{m}, \bar{h} \approx 3 \mu \mathrm{m})$, followed until steady state. Drawings (c) and (f) sketch $\mathbf{n}(\mathbf{r})$ for states (b) and (e). The focus is initially on the thin defect-rich shell side; thus, the first signs of the transition-starting on the thick side-are blurry (b). In (h) and (i) (no analyzer) the focus is on the thick shell side. 
texture, cf. Figs. 2(i), 3(f), 5(h), and 5(i). This modulation is again a result of a layer undulation instability, this time setting in after the initial stripe formation but before the lunes are fully developed, cf. Figs. 3(d) and 3(e). When the initial stripes form, this resolves the strain on the layers imposed by the spherical curvature in the direction parallel to $\mathbf{n}(\mathbf{r})_{N}$. The perpendicular curvature, along the layer, initially poses no problem since the internal structure of a single smectic layer has no positional order. However, as the layers buckle during the first stripe formation, n changes direction such that also the curvature perpendicular to $\mathbf{n}(\mathbf{r})_{N}$ would induce layer strain if no further adjustment took place. The system again resorts to layer buckling to solve the problem and this is what gives rise to the final stripe pattern decorating the lunes.

The $N$-SmA transition always begins opposite of the area where the defects are originally collected [Figs. 2(e) and 5(b)] and the smectic phase then grows around the shell, with the defect-rich area going through the phase transition last. This area is also the thinnest; i.e., here the problems of shell curvature are less severe. A greater problem is posed by the bend in $\mathbf{n}(\mathbf{r})$ within the shell plane, which is strong in the vicinity of the $s=1 / 2$ defects, cf. Figs. 2(b) and 5(c). To analyze this issue in more detail we now study the series of textures in Fig. 5. The shell here is somewhat thinner than that of Fig. 2, as evidenced by slightly stronger birefringence colors. In agreement with the results of Lopez-Leon et al. [2] the defects are then less strongly collected towards the point of minimum thickness. In fact, one defect is initially on the thick side, leaving the other three forming an equilateral triangle around the shell bottom [Fig. 5(a)]. While cooling towards the transition the fourth defect moves around to the thinner half and the other defects redistribute, leaving also this shell with a configuration of two defect pairs. Defects 1 and 3 are dominated by bend deformation [cf. Figure 5(c)] and are somewhat closer to each other and to the point of minimum shell thickness.

The transition to SmA on the thick top side of the shell is recognized as a diffuse horizontal patterning in Fig. 5(b) and in (d)-(e) we see the stripes along the shell perimeter. When finally the area between the defects turns smectic a new stripe pattern develops between the 1 and 3 defect pair [Figs. 5(d) and 5(e)], perpendicular to the pattern that originated on the other side. This pattern can also be explained as a result of a buckling instability, this time triggered by the bend in $\mathbf{n}(\mathbf{r})$ in the shell plane [Fig. 5(c)]. As illustrated in Fig. 5(f) the buckling accommodates the increasing apparent interlayer distance on going from the straight line between defects 1 and 3 to the curved boundaries of the domain defined by all four defects. Later on, the texture is gradually more broken up, eventually resembling a traditional fan-shaped texture of smectics [Fig. 5(g)].

During the phase transition (b) $-(\mathrm{g})$ the defects first retain their configuration, but after a slight delay the texture on the thinner defect-containing bottom side of the shell is somewhat annealed, and in the process the 1 and 3 defects move substantially further apart. As a consequence the distance between the defects is eventually quite similar in both pairs at low SmA temperatures, cf. Fig. 5(g). The four defects now approach a configuration where they are all situated on a great circle, as predicted for $K_{3} / K_{1} \rightarrow \infty$ [5], appropriate for the smectic case.

We have described and explained the consequences of smectic ordering on thin liquid crystalline shells with planar alignment. The frustration between the curvature and nonzero thickness of the shell, a locally nonuniform director field, and the well-defined smectic layer thickness leads to several instabilities, producing different types of regular texture. This is only the first example of a wide range of structure formation schemes that can be studied with smectic shells. Slight variations in material and/or phase type, alignment and shell geometry will give different results, opening for future investigations. In addition to being a fascinating playground for soft matter physics research the shells may have potential for applications in, e.g., colloid science, where the different regular structures may provide a basis for colloids with directed interactions [8], the higher degree of order possibly removing some of the difficulties encountered when using nematic shells [1].

Support from the German Academic Exchange Service, the "Excellenzcluster nanostrukturierte Materialien" (Sachsen-Anhalt), the Swedish Research Council and the AICT (SNU, Korea) is gratefully acknowledged.

*jan.lagerwall@1csoftmatter.com

[1] A. Fernandez-Nieves, V. Vitelli, A. S. Utada, D. R. Link, M. Marquez, D. R. Nelson, and D. A. Weitz, Phys. Rev. Lett. 99, 157801 (2007).

[2] T. Lopez-Leon, V. Koning, K. B. S. Devaiah, V. Vitelli, and A. Fernandez-Nieves, Nature Phys. 7, 391 (2011).

[3] T. Lopez-Leon and A. Fernandez-Nieves, Phys. Rev. E 79, 021707 (2009).

[4] G. Skacej and C. Zannoni, Phys. Rev. Lett. 100, 197802 (2008).

[5] H. Shin, M. J. Bowick, and X. Xing, Phys. Rev. Lett. 101, 037802 (2008).

[6] V. Vitelli and D. R. Nelson, Phys. Rev. E 74, 021711 (2006).

[7] M. Bates, G. Skacej, and C. Zannoni, Soft Matter 6, 655 (2010).

[8] D. Nelson, Nano Lett. 2, 1125 (2002).

[9] S. Samitsu, Y. Takanishi, and J. Yamamoto, Nature Mater. 9, 816 (2010).

[10] J. P. F. Lagerwall and F. Giesselmann, Chem. Phys. Chem. 7, 20 (2006).

[11] A. Utada, E. Lorenceau, D. R. Link, P. D. Kaplan, H. A. Stone, and D. A. Weitz, Science 308, 537 (2005).

[12] H.-L. Liang, E. Enz, G. Scalia, and J. Lagerwall, Mol. Cryst. Liq. Cryst. (to be published).

[13] P.-G. de Gennes and J. Prost, The Physics of Liquid Crystals (Clarendon Press, Oxford, UK, 1993).

[14] M. Kleman and O. D. Lavrentovich, Soft Matter Physics: An Introduction (Springer, New York, 2002).

[15] S. T. Lagerwall, Ferroelectric and Antiferroelectric Liquid Crystals (Wiley-VCH, Weinheim, 1999). 